\title{
BMJ Open Improving quality and safety in nursing homes and home care: the study protocol of a mixed-methods research design to implement a leadership intervention
}

\author{
Siri Wiig, ${ }^{1}$ Eline Ree, ${ }^{1}$ Terese Johannessen, ${ }^{1}$ Torunn Strømme, ${ }^{1}$ Marianne Storm, ${ }^{1}$ \\ Ingunn Aase, ${ }^{1}$ Berit Ullebust, ${ }^{2}$ Elisabeth Holen-Rabbersvik, ${ }^{1,3,4}$ \\ Line Hurup Thomsen, ${ }^{5}$ Anne Torhild Sandvik Pedersen, ${ }^{6}$ \\ Hester van de Bovenkamp, ${ }^{7}$ Roland Bal, ${ }^{7}$ Karina Aase ${ }^{1}$
}

To cite: Wiig S, Ree E, Johannessen T, et al. Improving quality and safety in nursing homes and home care: the study protocol of a mixedmethods research design to implement a leadership intervention. BMJ Open 2018;8:e020933. doi:10.1136/ bmjopen-2017-020933

- Prepublication history for this paper is available online. To view these files, please visit the journal online (http://dx.doi. org/10.1136/bmjopen-2017020933).

Received 4 December 2017 Revised 7 February 2018 Accepted 20 February 2018

Check for updates

For numbered affiliations see end of article.

Correspondence to

Professor Siri Wiig;

siri.wiig@uis.no

\section{ABSTRACT}

Introduction Nursing homes and home care face challenges across different countries as people are living longer, often with chronic conditions. There is a lack of knowledge regarding implementation and impact of quality and safety interventions as most research evidence so far is generated in hospitals. Additionally, there is a lack of effective leadership tools for quality and safety improvement work in this context.

Methods and analysis The aim of the 'Improving Quality and Safety in Primary Care-Implementing a Leadership Intervention in Nursing Homes and Homecare' (SAFELEAD) study is to develop and evaluate a research-based leadership guide for managers to increase quality and safety competence. The project applies a mixed-methods design and explores the implications of the leadership guide on managers' and staffs' knowledge, attitudes and practices. Four nursing homes and four home care services from different Norwegian municipalities will participate in the intervention. Surveys, process evaluation (interviews, observations) and document analyses will be conducted to evaluate the implementation and impact of the leadership intervention. A comparative study of Norway and the Netherlands will establish knowledge of the context dependency of the intervention.

Ethics and dissemination The study is approved by the Norwegian Centre for Research Data (2017/52324 and 54855). The results will be disseminated through scientific articles, two $\mathrm{PhD}$ dissertations, an anthology, presentations at national and international conferences, and in social media, newsletters and in the press. The results will generate knowledge to inform leadership practices in nursing homes and home care. Moreover, the study will build new theory on leadership interventions and the role of contextual factors in nursing homes and home care.

\section{INTRODUCTION}

\section{Quality and safety challenges}

Nursing homes and home care face challenges worldwide as people live longer,
Strengths and limitations of this study

- The study translates European Union research findings into practice by implementing a leadership-focused quality and safety improvement intervention in Norwegian nursing homes (4) and home care (4).

- A key strength of the Improving Quality and Safety in Primary Care-Implementing a Leadership Intervention in Nursing Homes and Homecare (SAFE-LEAD) study is user involvement in all phases, including coresearchers representing patients, next of kin, a patient and user ombudsman, and managers in nursing homes and home care.

- A mixed-methods design involving a contrasting case-study approach enables the SAFE-LEAD study to explore the role of context when implementing a leadership intervention in nursing homes and home care services located in large, small, rural and urban municipalities.

- Process evaluation over 12 months will be limited to four out of eight recruited institutions in the SAFELEAD intervention giving few possibilities for generalising results.

- Despite a detailed adaptive process with an extensive level of user involvement, building on a leadership guide initially developed for the hospital context might disregard information vital for the nursing home and home care services context.

often with one or more chronic conditions that should be treated as conservatively as possible. ${ }^{1}$ Most of the research on quality and safety in healthcare is conducted in hospital contexts so we know little about other healthcare settings. ${ }^{2}$ Numerous quality and safety challenges exist in the nursing home and home care contexts. For example, safety in home care is inseparable from relationships and interactions between patients, informal 
caregivers and formal healthcare providers. ${ }^{3-6}$ In addition, minor mistakes, discontinuity and multiple care providers with little overview of patient status and development may cause cumulative negative effects over time. This cumulative effect is especially important when we consider quality and safety at home and in the community. ${ }^{1}$ In this context, the role of organisational structures and processes is under-researched, and there is a need for more knowledge on the ways in which organisational and provider factors combine to affect quality and safety. ${ }^{27}$

\section{The role of management and leadership}

In recent work, Mintzberg ${ }^{8}$ focuses on the importance of leadership in healthcare management, as the two have often been separated. Management involvement and a wide range of leadership roles and activities are crucial in the development of structures and cultures to improve patient safety and achieve sustained quality in healthcare services. ${ }^{29-14}$ Although quality and safety improvement work in healthcare is predicated on interaction and collaboration among many organisational stakeholders, ${ }^{1115}$ the onus is on healthcare managers to commit to improvement efforts and use research-based knowledge in planning and improving quality and safety work. ${ }^{16-18}$ Involving managers in properly designed implementation programmes has been found to have a positive impact on organisational outcomes, ${ }^{19}$ especially if the programmes are comprehensive and systematically integrated into the organisational culture. ${ }^{20}$ As such, there is ongoing demand for more and better knowledge about quality and safety improvement work focused on the abilities and capacities of managers. ${ }^{13}$ Of particular concern is strengthening leadership capacity, competence, and quality and safety in nursing homes and home care services.

\section{Understanding the role of context in knowledge translation}

Translating research-based knowledge into practice in healthcare is challenging. ${ }^{212}$ One of the key challenges for management teams is how to implement evidencebased knowledge to facilitate quality and safety improvement at the local service level. ${ }^{15}{ }^{23}$ Many knowledge translation frameworks have been proposed that acknowledge the socially situated nature of knowledge implementation practices. ${ }^{22}{ }^{24-26}$ Nevertheless, the literature says little about the influence of context on successful quality and safety implementation interventions in healthcare. ${ }^{2327-30}$ Context can refer to both the inner (internal) and outer (external) settings of an organisation. Internal organisational factors include structural characteristics (eg, location and size); the local workings of teams and leadership; and the organisational culture and implementation climate. Among the external factors are applicable laws, regulatory requirements, external policies and incentives, and funding structures. ${ }^{29}$ Differences in internal and external organisational contexts are thought to be responsible for some of the variability seen in the implementation of quality and safety improvement efforts in diverse local practice settings. ${ }^{28}{ }^{29}$ However, there are few if any studies of the role of contextual factors in leadership interventions in nursing homes and home care.

\section{Organisation and quality and safety status of primary care in Norway}

Although the organisation and responsibilities of healthcare systems can differ, the primary care setting is an important arena for the provision of health and social care services in Norway as in many other countries. In Norway, the delivery of primary care services is the responsibility of the municipalities, which provide most of the country's home care and nursing home services, which is within the scope of our study. The Norwegian municipalities are by law required to work to improve healthcare quality and safety, and managers at all service levels are responsible for the planning, implementation, evaluation and systematic improvement of service quality and safety.

However, as noted in a recent government white paper on Norwegian primary care services, quality and safety efforts in municipal health services to date have been insufficient. ${ }^{31}$ Inspections made by the Norwegian Board of Health Supervision have repeatedly found inadequate quality assurance and control measures across primary care organisations. While quality improvement and safety efforts should be a top priority of municipal management teams, quality and safety work is often poorly rooted in management, and in some places not considered a management task at all. ${ }^{32}$ Where quality and safety improvement work has been undertaken, there have been challenges in translating knowledge into practice. ${ }^{31}$ Such reports have led to the conclusion that there is a serious lack of basic leadership competencies related to quality and safety improvement work in Norwegian primary care services. The latter constitutes the major rationale for conducting our study.

\section{Aims and research questions}

The 'Improving Quality and Safety in Primary CareImplementing a Leadership Intervention in Nursing Homes and Homecare' (SAFE-LEAD) study is translating research findings from the European Union seventh Framework Programme funded project 'Quality and Safety in Europe by Research' (QUASER) into practice in Norway by implementing a leadership-focused quality and safety improvement intervention in the nursing homes and home care settings. The intervention is built around the implementation of a quality and safety improvement tool, which is a leadership guide for managers in nursing homes and home care. The leadership guide is based on the results from the QUASER study ${ }^{33}$ where the consortium, including SAFE-LEAD partners, developed the QUASER Hospital Guide-a research-based tool to reflect on and develop your quality improvement strategies. ${ }^{34}$ The QUASER Hospital Guide defines quality as care that is clinically effective, safe and patient centred. The guide is structured around eight quality challenges (structure, culture, leadership, politics, education, emotions, 
physical and technical issues, external demands). A short series of questions will stimulate reflection, accompanied by a decision-aid menu of potential options, with empirical examples of possible quality and safety improvement solutions across the macro, meso and micro system levels. The guide is designed to facilitate patient safety and quality improvement in clinical practice and service delivery, by providing a systematic means for managers to pinpoint the strengths and weaknesses of their improvement strategies and reflect on what the tailored measures needed in their institution and context.

The SAFE-LEAD study will investigate how and to what extent different contextual factors influence the implementation process and the effectiveness of such a research-based guide in a variety of nursing homes and home care services. The aim of the study is to build leadership competence and guide managers in their efforts to advance and improve vital quality and safety strategies, attitudes and practices in their organisations. The specific objectives of the SAFE-LEAD study are to:

a. Investigate the influence of context on the implementation of a research-based quality and safety leadership intervention in nursing homes and home care.

b. Test the effectiveness of the leadership intervention on changes in managers' and healthcare professionals' knowledge, attitudes and practices relating to quality and safety in nursing homes and home care.

c. Develop theory to guide implementation of future leadership interventions designed to improve the quality and safety in nursing homes and home care.

The following research questions will guide the SAFELEAD study:

1. What are the key contextual factors that affect quality and safety improvement work in the Norwegian nursing homes and home care setting?

2. How can the SAFE-LEAD intervention best be designed to implement use of a leadership guide in nursing homes and home care?

3. Which contextual factors, including leadership practices and processes influence successful implementation and use of a leadership guide in nursing homes and home care?

4. How can patient and next-of-kin involvement be integrated into use of a leadership guide and the overall SAFE-LEAD intervention?

5. What is the impact of the SAFE-LEAD intervention on managers' and staffs' quality and safety knowledge, attitudes, and practice?

6. What are the implications of the SAFE-LEAD research findings on the development of theoretical frameworks for organisational context, leadership processes, and quality and safety improvement efforts in nursing homes and home care settings?

7. What are the similarities and differences in contextual factors determining successful implementation of research-based quality and safety improvement tools in Norway and the Netherlands?
8. How and to what extent do the identified key contextual factors explain implementation, uptake and impact of the SAFE-LEAD intervention across nursing homes and home care services?

\section{METHODOLOGY}

\section{Design}

The SAFE-LEAD study (2016-2021) applies a convergent parallel mixed-methods design. ${ }^{35}$ We will collect both quantitative and qualitative data in parallel, analyse them separately and compare results subsequently. ${ }^{35}$

\section{Setting}

The main study setting is nursing homes and home care in the Norwegian primary care system. In addition, a small-scale study will take place in a nursing home and a home care institution in the Netherlands.

\section{Study sample and recruitment}

Four nursing homes and four home care services from different Norwegian municipalities will be recruited to participate in the SAFE-LEAD intervention. To understand the role of context, the sampling strategy is based on a contrasting case approach, ${ }^{36}$ with selection criteria focusing on diversity in size, geography and variation between urban and rural services. A similar small-scale study of one nursing home and one home care service will be conducted in the Dutch healthcare setting, allowing for comparison of two countries with different national healthcare and regulatory systems. The recruitment of Norwegian institutions will be conducted in collaboration with two Centres for Development of Institutional and Home Care Services (Rogaland County, Sogn and Fjordane County) and the municipality of Songdalen in Vest-Agder County. The Dutch research team will recruit the institutions in the Netherlands.

\section{Data collection methods and sources}

The study is structured around five work packages (WPs) indicating distinct phases of the project. In the following, we describe the phases, data collection and sources.

Phase 1: guide development, pilot test and contextual mapping tool (WP1)

In phase 1, we will develop the quality and safety improvement tool-the SAFE-LEAD guide - to be used in the SAFE-LEAD intervention. This includes translation and adaptation of the QUASER Hospital Guide in to Norwegian in a process involving the research team in several iterations with professional translation services, coresearchers, future users, and patient and next-of-kin representatives. The original QUASER guide is based on empirical findings from the hospital setting and the SAFE-LEAD project will develop a version adapted to the Norwegian nursing homes and home care setting. The guide will be in one version similar for both nursing homes and home care. Possible challenges in adapting and further developing a tool, that is, originally developed for hospital managers, 
include different management contexts, tasks, resources, knowledge level among healthcare staff, larger variety in institutional size, resources and management levels. Therefore, the SAFE-LEAD study has designed a comprehensive development process over the first year in the project period. This development process will consist of internal workshops with the multidisciplinary research team with competence in nursing, home care, nursing homes, quality and safety, leadership, health promotion, and human factors. There will also be workshops with coresearchers in the SAFE-LEAD partner consortium ensuring sound user involvement with perspectives from patient representatives, next-of-kin representatives, a patient and user ombudsman, and perspectives from future users of the guide (managers in primary care). To ensure that the guide fits the new context, we will conduct three to four focus group interviews to collect input from managers in nursing homes and home care services who will have read the guide beforehand. Finally, the guide will be tested for fit and validity in one nursing home and one home care service with senior healthcare managers and their teams as part of the pilot test of the intervention (described in phase 2). The two pilot institutions are not part of the sample of eight institutions that will be recruited for the full intervention in phase 3 .

To facilitate implementation and use of the guide, we will also offer a web-based version. The web version will have the same content as the paper version and both the paper and the web version will be published and publicly available for all Norwegian healthcare services, who may find it relevant, on a SAFE-LEAD website after completion of the project in 2021.

To assess the influence of contextual factors, we will develop a mapping tool for use in the implementation and evaluation phase of the project. This will be inspired by (1) Damschroder et $a l \mathrm{~s}^{25}$ Consolidated Framework for Implementation Research (CFIR) and McDonald's ${ }^{29}$ framework for considering context in quality and safety improvement interventions, (2) additional literature searches and (3) a qualitative study with 10-12 nursing home and home care managers in a variety of Norwegian municipalities (large, small, rural, urban). In the interviews, we will map the contextual factors of relevance for managers' work on quality and safety in nursing homes and home care. The interview guide includes open questions regarding which factors managers perceive as important for their work with quality and safety, and topics such as external factors, economy and structure. The tool will consider factors such as type of healthcare service (nursing home or home care), funding, geographical location, organisation size, workload and any ongoing national/regional/organisational change processes.

Phase 2: intervention design, pilot testing and recruitment (WP2) The Medical Research Council's guidance on developing, testing and evaluating complex interventions to improve healthcare ${ }^{37} 38$ will be used to design the intervention. This framework views healthcare interventions as flexible, non-linear processes, giving equal attention to all process phases (development, testing, evaluation, wider application). Furthermore, it stresses the importance of context in implementation and allows an intervention to be adapted to its setting, to better ensure its success in practice. ${ }^{373}$ The application of an organisational perspective has been suggested as an aid to understanding the contextual factors and processes that may enable or impede knowledge implementation interventions in healthcare settings. ${ }^{29} 39$ As the SAFE-LEAD study is concerned with implementation in practice, the Organising for Quality framework ${ }^{1140}$ will be used as a theoretical foundation in the intervention design, alongside the CFIR. ${ }^{25}$ Both frameworks advocate a multilevel contextual perspective on the implementation and evaluation of interventions.

\section{The SAFE-LEAD intervention}

The SAFE-LEAD intervention will be conducted in two stages over a period of 1 year. ${ }^{37}$ Stage 1 is a training component involving action learning workshops in which managers and their teams will be able to apply the guide and conduct a self-diagnosis of their current quality and safety work. A team of experienced researchers will facilitate reflexive group discussions among the teams, which will take place in four group sessions (2-3 hours each) in all participating institutions over a 6 -month period. It is proposed that each group will consist of an extended management team (director of health and care services in municipality, nursing home director/director of home care services, department managers, head nurses, nursing home physicians and patient or next-of-kin representatives). These sessions will:

1. Introduce the guide to the participating institutions. This includes rationale, concepts, web tool and procedure for an internal management process for use of the guide within the organisation.

2. Provide guidance for integrating patient and next-ofkin experiences in quality and safety improvement work.

3. Establish strategies to address the diagnosed quality and safety challenges.

In stage 2 of the intervention, a sample of two nursing homes and two home care services (from the total sample of eight institutions) will receive a more comprehensive intervention component consisting of a close collaboration with the researchers. In addition to the training component described in stage 1 , the institutions in stage 2 will receive three site visits by researchers (1-3 days) per institution over a period of 12 months (stage $1+$ stage $2=12$ months). Activities during site visits will include:

1. A workshop to support existing and new learning arenas in quality and safety improvement work (1 day).

2. Observation of and feedback on quality and safety leadership strategy and practices (3 days).

3. A workshop to support the integration of patient and next-of-kin experiences in improvement work (1 day).

A pilot test of the stage 1 intervention will be conducted in one nursing home and one home care services setting 
(not part of the sample in the full intervention in phase 3) over a 3-month period to test the chosen intervention contents, pedagogical approaches and the functionality of the guide developed in phase 1 . We will evaluate the pilot intervention components by means of a qualitative process evaluation ${ }^{37} 38$ involving observation in the workshops and semistructured interviews with pilot intervention participants (15-20). The pilot will not test the outcome measures. The intervention will be tailored, based on the pilot results.

Phase 3: testing and evaluating the SAFE-LEAD intervention (WP3) In phase 3, we will implement the intervention stages 1 and 2. Before and during the implementation process, we will map contextual factors in all participating institutions by using the context mapping tool developed in phase 1 .

The SAFE-LEAD intervention is centred on the testing in practice of a research-based quality and safety improvement guide for managers. The Knowledge-to-Action framework ${ }^{22}$ will be used to guide this part of the intervention. This approach proposes that the translation into practice of a research-based guide requires an organisation to identify the problems it needs to solve; adapt the guide to its own settings and contexts; assess and address barriers to its use; implement the intervention; monitor the implementation and evaluate the outcomes. ${ }^{42}$ The contents and procedures involved in this phase of the SAFE-LEAD intervention will be designed and developed in close collaboration with the participating user representatives in the research team, based on a reflexive, dialogue-based group technique.

The evaluation of the study entails both an in-depth qualitative work to understand the process of implementing the quality and safety improvement guide in practice; how the participating nursing homes and home care institutions use the guide and a quantitative measurement of the impact of the guide on quality and safety improvement knowledge and practices in these institutions.

Although the SAFE-LEAD intervention is a leadership intervention designed to address leadership issues, knowledge must be disseminated and applied at the clinical level (eg, nurses and doctors) for the implementation to be effective. In the evaluation of stage 1 , we will assess the relationship between using the guide and changes in staffs' and managers' quality and safety knowledge, attitudes and practices in all eight participating institutions. We will measure this relationship by using a knowledge, attitude and practice (KAP) survey. We will conduct a literature review to identify and select measurement scales for the survey questionnaire. Managers and staff in all participating institutions will be invited to respond to the baseline survey questionnaire before the intervention starts. A second survey questionnaire will be administered to the study participants after the stage 1 intervention (after 6 months). The rationale is to measure changes in quality and safety knowledge, attitudes and practices following the intervention programme stage 1 .

The purpose of intervention stage 2 is to establish learning arenas, structures and processes to support leaders' self-diagnosis of their quality and safety work, and strengthen their capacity to conduct future improvement without researcher involvement. To evaluate the intervention stage 2 , we will conduct a process evaluation. ${ }^{38}{ }^{41}$ The process evaluation will require active researcher involvement in the intervention workshops. Data collection to evaluate the intervention processes in each of the four institutions (stage 2) will comprise semistructured interviews before and after the intervention period (over 12 months) with managers (approximately $5-10 \times 2$ depending on institution size) and staff (approximately 8-10×2 depending on institution size), observation of the intervention workshops and daily practice situations in the selected institutions (40-50hours), as well as document analysis of strategies, plans and regulatory inspection reports. During the intervention period, we will also conduct short follow-up conversations with managers participating in the intervention to collect information for use in the observation and feedback sessions. This implies a total of approximately 160 semistructured interviews and 150-200hours of observation. Interview guides and observation guides have been developed for managers and healthcare professionals. These guides are based on Bate $e t a l^{11}$ and cover quality and safety challenges in terms of culture, structure, enthusiasm, education, politics, external demands, physical and technical aspects, use of diverse tools in improvement work, and changes to these factors over time at the managerial and healthcare professional level. The study will apply NVivo to structure, categorise and analyse the qualitative data according to categories relevant for quality and safety improvement challenges. ${ }^{11}$ Data will be analysed within cases before conducting the cross-case comparison. $^{43}$

Across phase 1-3: tracer project on quality improvement in home care (WP4)

To arrive at an in-depth understanding of the role of context and leadership in daily quality improvement work, we will include a tracer quality improvement project as part of the data collection. ${ }^{33}$ The tracer project will be studied longitudinally over the entire project period with a combination of qualitative interviews, document analysis, observation and shadowing of staff. In the tracer project, we will evaluate an improvement project titled 'ABCDE-systematic observation and communication in community healthcare'. The improvement project aims at (1) developing a tailored educational programme designed to improve healthcare professionals' competencies and skills in recognising and responding to deteriorating frail patients and (2) implementing new work routines in the home care organisations to strengthen healthcare professionals' understanding and clinical judgement of deteriorating patients. The tracer project is identified with the project partners and is initiated by one of the partner municipalities. The SAFE-LEAD study will examine improvement processes in real time as exemplars of how quality improvement is implemented. The tracer project will enable lessons learnt, and contribute to understanding how managers are improving the professional observational skills of their staff in the home care context. 
Phase 4: mixed-methods synthesis, cross-country comparison, theory development (WP5)

The SAFE-LEAD study is an opportunity to understand the meaning and impact of contextual factors by analysing the influence of the quality and safety improvement guide implementation via three data sets: (1) a quantitative survey of outcome measures (knowledge, attitudes, practice) across a sample of eight institutions, (2) a context mapping of all participating institutions and (3) a qualitative multiple case study with a smaller sample of four institutions providing rich information on leadership processes and practices, a small-scale implementation case study in the Netherlands and a tracer quality improvement project. In this sense, the study pays closer and more explicit attention to multiple contextual factors, ${ }^{445}$ and the way in which they affect the success and sustainability of implementing quality and safety improvement tools.

The different data sets will be collected in parallel and analysed separately. ${ }^{35}$ In phase 4 of the study, we will synthesise the results from the qualitative, quantitative and context data sets by using a procedure called 'joint display of data'. The researchers will jointly display all forms of data (eg, in tables or in NVivo) responding to similar concepts and research questions. The analysis of the total amount of data in the Norwegian part of the study will compare the results from the concepts measured in the KAP survey, and what is mapped by qualitative process evaluation methods and the findings from the tracer project. The outcomes of the analyses will be used to develop theoretical frameworks and conceptual models of the influence of context and leadership on quality and safety improvement work in primary care settings.

The Norwegian results will then be compared with Dutch results from a similar small-scale research project, focusing on managers' use of different quality and safety improvement tools in general (not the SAFE-LEAD guide in specific). The Dutch cases are used to contrast the nursing homes and home care in Norway, as the Dutch healthcare system frequently uses a greater range of improvement tools as part of managing quality and safety than the Norwegian. The

Table 1 Planned primary publications from the SAFE-LEAD study

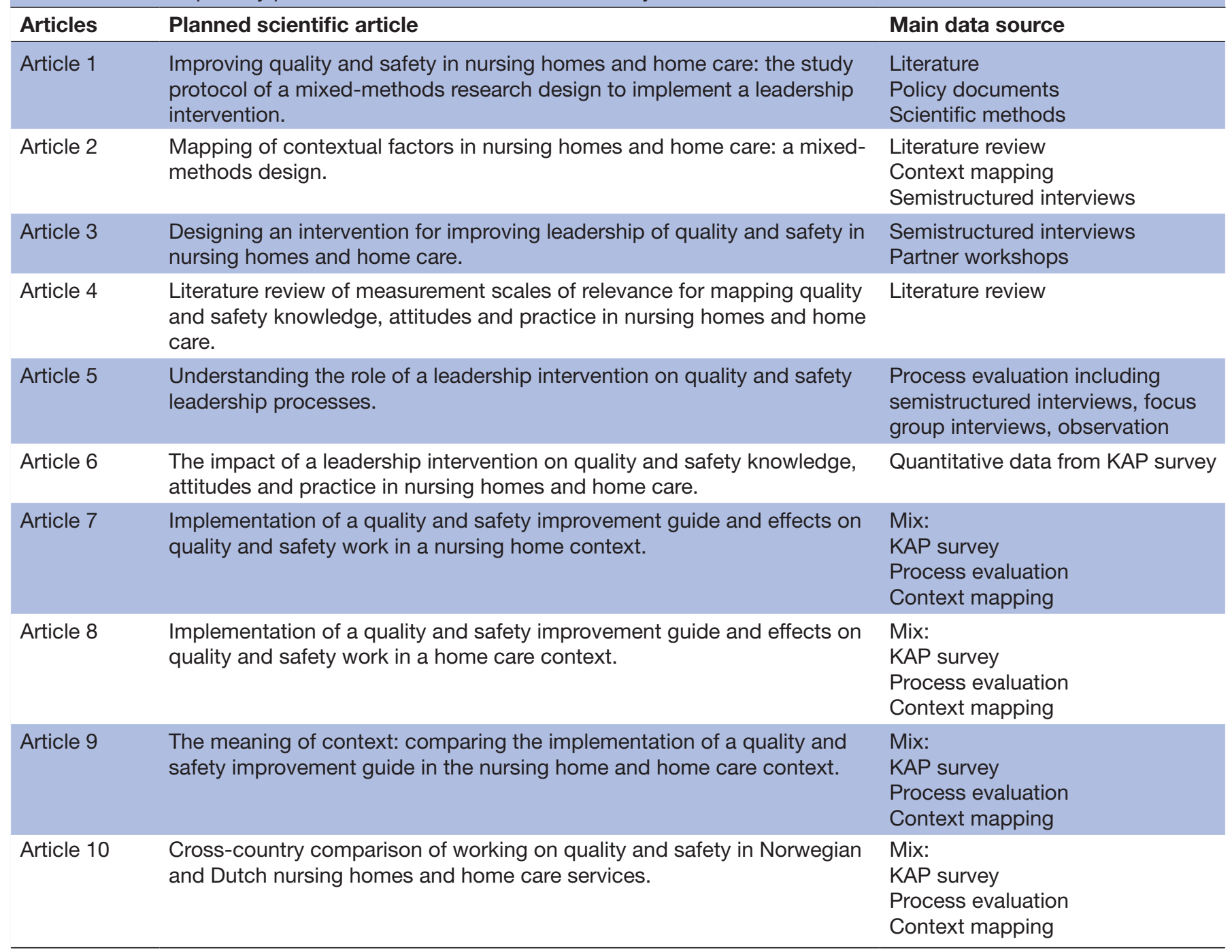


cross-country analysis will compare and contrast managers' practice and competence, and whether staff members make changes in their work practice to improve quality and safety of service provision. The comparison will build on a contrasting case approach ${ }^{43}$ based on the differences between the organisation of quality and safety work in healthcare systems in the two countries. Building on the approach taken in the QUASER study, ${ }^{33}$ a multilevel perspective will be used, considering important macro-level contextual factors (national healthcare system), in addition to the factors identified at the meso and micro levels in the case studies. We will look at effects of different contextual factors such as funding frameworks, regulation, prioritisation, organisation and competence level. National differences will be analysed to better understand the effect of macro-level healthcare system factors on the success of quality improvement implementation processes.

\section{ETHICS AND DISSEMINATION Ethical reflections}

The SAFE-LEAD leadership-focused intervention targets a potential knowledge gap among managers and supports their work on quality and safety improvement. The risk of negative effects on patient outcomes is thus minimal. The potential of not having a positive effect of the intervention on managers' leadership competence is present, but the risk of negative patient outcome due to this is limited. The patient and nextof-kin perspectives are key throughout the SAFE-LEAD study. Patients and next-of-kin representatives participated in the project development and will collaborate with the project team as coresearchers throughout the project period. Different measures are involved in the quality and safety improvement guide itself (strategies, measures) and in the intervention components (workshops on use of patient experiences) to improve managers' abilities to involve patient and next of kin in improvement work.

No health information will be collected. The Norwegian approval also includes approval for data collection in the Netherlands. The Dutch ethical approval system does not require ethical approval for research projects not involving patient data, as in the SAFE-LEAD study. All participants will sign informed consent and will be recruited on a voluntary basis. No patient records or other patient data will be collected.

\section{Dissemination}

The SAFE-LEAD partners have agreed on publication guidelines, a publication strategy and a publication plan. The publication strategy consists of dissemination in scientific peer-reviewed journals, books and presentations at academic conferences. Moreover, there is a strategy to ensure dissemination in popular science forums and in social media. The project has established a SAFE-LEAD web page, a SAFE-LEAD Facebook account, newsletters and posters. The project has an international expert advisory board that will be invited annually to give input to the study and contribute to the sharing of results.

The publication plan will evolve over time but includes the following planned scientific dissemination activities:

- Two PhD theses (authors TJ and TS).

- Eighteen to twenty scientific articles in a peer-reviewed special issue and in different peer-reviewed journals.

- A book on Quality and Safety in Primary Care involving international contributions.

- Local seminars at the study sites (including user groups).

- Norway-Netherland seminar in 2020 to present final project results. Open seminar with target group healthcare managers, inspection authority, researchers, user groups, professional associations, governmental bodies, etc.

- Special sessions at the fifth and sixth Nordic Conference on Research in Patient Safety and Quality in Healthcare.

- Presentations at regional, national and international conferences.

- Popular science presentations in national media and healthcare magazines.

Ten primary publications are planned (see table 1):

\section{Author affiliations}

${ }^{1}$ SHARE- Centre for Resilience in Healthcare, Faculty of Health Sciences, University of Stavanger, Stavanger, Norway

${ }^{2}$ Center for Developing Institutional and Home Care Services Sogn and Fjordane,

Førde, Norway

${ }^{3}$ Department of Health and Nursing Sciences, University of Agder, Kristiansand,

Norway

${ }^{4}$ Songdalen Municipality, Songdalen, Norway

${ }^{5}$ Center for Developing Institutional and Home Care Services Rogaland, Stavanger,

Norway

${ }^{6}$ Next-Of-Kin Representative, Stavanger, Norway

${ }^{7}$ School of Health Policy \& Management, Erasmus University, Rotterdam, The Netherlands

Acknowledgements Siri Wiig, Karina Aase and Veslemøy Guise were responsible for the application for funding to the Research Council of Norway (RCN). We wish to acknowledge the contribution from Veslemøy Guise who played a key role in developing the grant application to the RCN, and to the Patient Ombudsman Vestfold Torunn Grinvoll, senior representative Elsa Kristiansen and Lene Schibevaag in the SAFE-LEAD project who have provided input to the study design and development. We wish to acknowledge the other members of the QUASER team: Naomi Fulop (project manager),Susan Burnett, Glenn Robert, Janet Anderson, Charles Vincent, Kathryn Charles, Susie Edwards, Lisbeth Hoeg-Jensen, Heidi Poestges and Anna Renz (England); Julia Quartz and Anne Marie Weggelaar (the Netherlands); Boel Anderson-Gäre, Pär Höglund, Tony Andersson, Anette Karltun, Johan Calltorp and Johan Sanne (Sweden); Francisco Nunes, Sara Gomes and Alexandra Fernandes (Portugal); Christian von Plessen (Norway). The authors would like to thank the two reviewers, Gunnar Tschudi Bondevik and Christine W.Hartman, for their valuable comments and input to improve the SAFE-LEAD study protocol.

Contributors SW and KA applied for funding of the SAFE-LEAD study to the Research Council of Norway, (RCN), planned the study design and study protocol, and contributed to the development of the data collection tools. SW and ER drafted the manuscript, with substantial input from KA, and revised it based on comments from all coauthors. ER contributed to the study design, development of data collection tools, and was responsible for the application for approval of the study to the Norwegian Centre for Research Data. Authors TS, TJ and MS contributed to the study design and development of data collection tools, and commented on the draft. Authors HB and RB contributed to the study design and development of the data collection tools, commented on the draft, and are responsible for the Dutch part of the study. Authors IA, BU, EH-R, LHT and ATSP contributed to the study design and 
commented on the draft. All authors approved the final version of the manuscript. Authors RB, HB, KA and SW were all part of the QUASER project from Norway and the Netherlands and contributed to writing the original guide.

Funding This work was supported by The Research Council of Norway (RCN) grant number 256681/H10 and the University of Stavanger, Norway. The leadership guide applied in the SAFE-LEAD study is based on the results from the study Quality and Safety in European Union Hospitals: A Research-based Guide for Implementing Best Practice and a Framework for Assessing Performance (QUASER). The QUASER project received funding from the European Community's Seventh Framework Programme (FP7/2007-2013) under grant agreement no.241724.

Competing interests None declared.

Patient consent Not required.

Ethics approval The SAFE-LEAD study is approved by the Norwegian Centre for Research Data (2017/52324 and 54855), and exempted from ethical approval from Regional Ethical Committee.

Provenance and peer review Not commissioned; externally peer reviewed.

Open Access This is an Open Access article distributed in accordance with the Creative Commons Attribution Non Commercial (CC BY-NC 4.0) license, which permits others to distribute, remix, adapt, build upon this work non-commercially, and license their derivative works on different terms, provided the original work is properly cited and the use is non-commercial. See: http://creativecommons.org/ licenses/by-nc/4.0/

(c) Article author(s) (or their employer(s) unless otherwise stated in the text of the article) 2018. All rights reserved. No commercial use is permitted unless otherwise expressly granted.

\section{REFERENCES}

1. Vincent C, Amalberti R. Safer healthcare. London: Springer Open, 2016.

2. Jha AK, Prasopa-Plaizier N, Larizgoitia I, et al. Patient safety research: an overview of the global evidence. Qual Saf Health Care 2010;19:42-7.

3. Henriksen $\mathrm{K}$, Joseph A, Zayas-Cabán T. The human factors of home health care: a conceptual model for examining safety and quality concerns. J Patient Saf 2009;5:229-36.

4. Macdonald MT, Lang A, Storch J, et al. Examining markers of safety in homecare using the international classification for patient safety. BMC Health Serv Res 2013;13:191.

5. Lang A, Edwards N, Fleiszer A. Safety in home care: a broadened perspective of patient safety. Int J Qual Health Care 2008;20:130-5.

6. Guise V, Anderson J, Wiig S. Patient safety risks associated with telecare: a systematic review and narrative synthesis of the literature. BMC Health Serv Res 2014;14:588.

7. Wooldridge AR, Carayon P, Hundt AS, et al. SEIPS-based process modeling in primary care. Appl Ergon 2017;60:240-54.

8. Mintzberg $\mathrm{H}$. Managing the myths of health care: bridging the separations between care, cure, control, and community: BerrettKoehler Publishers, 2017.

9. Oldenhof L, Stoopendaal A, Putters K, et al. From boundaries to boundary work: middle managers creating inter-organizational change. J Health Organ Manag 2016;30:1204-20.

10. Parand A, Dopson S, Renz A, et al. The role of hospital managers in quality and patient safety: a systematic review. BMJ Open 2014;4:e005055.

11. Bate P, Mendel P, Robert G. Organizing for quality: the improvement journeys of leading hospitals in Europe and the United States: Radcliffe Publishing, 2008.

12. Leape L, Berwick D, Clancy C, et al. Transforming healthcare: a safety imperative. Qual Saf Health Care 2009;18:424-8.

13. Künzle B, Kolbe M, Grote G. Ensuring patient safety through effective leadership behaviour: A literature review. Saf Sci 2010;48:1-17.

14. Glickman SW, Baggett KA, Krubert CG, et al. Promoting quality: the health-care organization from a management perspective. Int J Qual Health Care 2007;19:341-8.

15. Wiig S, Storm M, Aase K, et al. Investigating the use of patient involvement and patient experience in quality improvement in Norway: rhetoric or reality? BMC Health Serv Res 2013;13:206.

16. Ministry of Health and Care Services. Meld.St. 10 (2012-2013). God kvalitet - trygge tjenester - Kvalitet og pasientsikkerhet $i$ helse- og omsorgstjenesten. Oslo, 2012.

17. Ministry of Health and Care Services. Meld. St. 11 (2014-2015). Kvalitet og pasientsikkerhet 2013. Oslo, 2014.
18. Wiig S, Robert G, Anderson JE, et al. Applying different quality and safety models in healthcare improvement work: boundary objects and system thinking. Reliability Engineering \& System Safety 2014;125:134-44.

19. Collins DB, Holton EF. The effectiveness of managerial leadership development programs: a meta-analysis of studies from 1982 to 2001. Human Resource Development Quarterly 2004;15:217-48.

20. Amagoh F. Leadership development and leadership effectiveness. Management Decision 2009;47:989-99.

21. Tsoukas $\mathrm{H}$. The firm as a distributed knowledge system: a constructionist approach. Strategic Management Journal 1996;17:11-25.

22. Straus S, Tetroe J, Graham ID. Knowledge translation in health care: moving from evidence to practice. 2nd edn. Wiley Blackwell: Oxford, 2013.

23. Øvretveit J. Understanding the conditions for improvement: research to discover which context influences affect improvement success. BMJ Qual Saf 2011;20(Suppl 1):i18-i23.

24. Stetler CB, Ritchie JA, Rycroft-Malone J, et al. Institutionalizing evidence-based practice: an organizational case study using a model of strategic change. Implement Sci 2009;4:78.

25. Damschroder LJ, Aron DC, Keith RE, et al. Fostering implementation of health services research findings into practice: a consolidated framework for advancing implementation science. Implement Sci 2009;4:1.

26. Rycroft-Malone J, Bucknall T. Models and frameworks for implementing evidence-based practice: linking evidence to action. Wiley Blackwell: Oxford, 2010.

27. Shekelle PG, Pronovost PJ, Wachter RM, et al. Advancing the science of patient safety. Ann Intern Med 2011;154:693-6.

28. Kaplan HC, Brady PW, Dritz MC, et al. The influence of context on quality improvement success in health care: a systematic review of the literature. Milbank Q 2010;88:500-59.

29. McDonald KM. Considering context in quality improvement interventions and implementation: concepts, frameworks, and application. Acad Pediatr 2013;13:S45-S53.

30. Coles $\mathrm{E}$, Wells M, Maxwell M, et al. The influence of contextual factors on healthcare quality improvement initiatives: what works, for whom and in what setting? Protocol for a realist review. Syst Rev 2017;6:168

31. Ministry of Health and Care Services. Meld. St. 26 (2014-2015) Fremtidens primærhelsetjeneste - nærhet og helhet. Oslo, 2014.

32. Norwegian Board of Health Supervision. Tilsynsmelding 2013. Oslo, 2014.

33. Robert GB, Anderson JE, Burnett SJ, et al. A longitudinal, multi-level comparative study of quality and safety in European hospitals: the QUASER study protocol. BMC Health Serv Res 2011;11:285.

34. Fulop N. Quaser. The Hospital Guide. A research-based tool to reflect on and develop your quality improvement strategies: University College London. 2013 https://www.ucl.ac.uk/dahr/pdf/ study_documents/iQUASER_Hospital_Guide_291014_press-ready cs4.pdf (cited 13 Nov 2017)

35. Creswell JW. Research design: qualitative, quantitative, and mixed methods approaches: Sage publications, 2013.

36. Yin R. Case study research: design and methods. 5th edn: Thousand Oaks Sage, 2014.

37. Craig P, Dieppe P, Macintyre S, et al. Developing and evaluating complex interventions: the new medical research council guidance. BMJ 2008;337:a1655.

38. Moore GF, Audrey S, Barker M, et al. Process evaluation of complex interventions: Medical Research Council guidance. BMJ 2015;350:h1258.

39. Denis J-L, Lehoux P. Organizational theory. In: Straus S, Tetroe J, Graham I, eds. Knowledge translation in health care: moving from evidence to practice. Oxford: Wiley Blackwell, 2009.

40. Bergeroed I, Wiig S. Ledelse og pasientsikkerhet. (Leadership and patient safety). Aase K, eds. Pasientsikkerhet-teori og praksis. 2nd edn. Oslo: Universitetsforlaget, 2015.

41. Patton M. Qualitative research and evaluation methods. 3rd edn. Thousand Oaks: Sage, 2002.

42. Harrison MB, Graham ID, Fervers B, et al. Adapting knowledge to a local context. In: Tetroe J, Graham I, eds. Knowledge translation in health care: moving from evidence to practice. Oxford: Wiley Blackwell, 2009:73-82.

43. Yin R. Case study research: design and methods. 3rd edn. Thousand Oaks: Sage, 2003.

44. House R, Rousseau DM, Thomas-Hunt M. The meso paradig: a framework for the integration of micro and macro organizational behavior. Review of Organization Behavior 1995;17:71-114.

45. Wiig S. Contributions to risk management in the public sector. PhD Thesis UiS no 48 Feb 2008. 